\title{
LncRNA THRIL is upregulated in sepsis and sponges miR-19a to upregulate TNF-a in human bronchial epithelial cells
}

\author{
Tao Liu ${ }^{1 \dagger}$, Jingbin Liü ${ }^{2 \dagger}$, Chunhua Tian ${ }^{3 *}$, Hongyuan $\mathrm{Wang}^{4}$, Min Wen ${ }^{5}$ and Mingyu Yan ${ }^{1}$
}

\begin{abstract}
Background: Long non-coding RNAs (IncRNAs) have been demonstrated to play critical roles in various diseases. Our bioinformatics analysis showed that IncRNA TNFa and heterogenous nuclear ribonucleoprotein $L$ (hnRNPL) related immunoregulatory LincRNA (THRIL) may interact with miR-19a, which targets TNF-a. This study aimed to explore the role of THRIL, an enhancer of LPS-induced inflammatory, in sepsis.
\end{abstract}

Methods: Research subjects of the present study included 66 sepsis patients and 66 healthy volunteers. The expression levels of THRLL, miR-19a and TNF-a in plasma samples from these participants were determined by RT-qPCR. The interaction between THRLL and miR-19a was explored by performing overexpression experiments in human bronchial epithelial cells (HBEpCs). The roles of THRIL, miR-19a and TNF-a in regulating the apoptosis of HBEpCs were analyzed by cell apoptosis assay.

Results: We found that THRIL was upregulated in sepsis patients. THRIL is predicted to interact with miR-19a, and the interaction was confirmed by dual-luciferase activity assay. However, THRIL and miR-19a did not affect the expression of each other. Instead, overexpression of THRIL resulted in the increased expression levels of TNF-a, a downstream target of miR-19a in HBEpCs. In HBEpCs, LPS treatment induced the overexpression of THRIL. Cell apoptosis analysis showed that overexpression of THRIL and TNF-a promoted the apoptosis of HBEpCs induced by LPS, while overexpression of miR-19a played an opposite role. Overexpression of THRIL attenuated the effects of overexpression of miR-19a.

Conclusion: Therefore, THRIL is upregulated in sepsis and may sponge miR-19a to upregulate TNF-a, thereby promoting lung cell apoptosis.

Keywords: Sepsis, IncRNA THRIL, miR-19a, TNF-a, Apoptosis

\section{Background}

Sepsis is the body's extreme reactions to infection that can result in organ failure, tissue damage or even death if treatment was not performed properly and timely [1]. In severe cases, mortality rate caused by sepsis may

\footnotetext{
* Correspondence: zw9390@163.com

${ }^{\dagger}$ Tao Liu and Jingbin Liu contributed equally to this work.

${ }^{3}$ Department of Nephrology, Inner Mongolia Baogang Hospital (The Third Affiliated Hospital of Inner Mongolia Medical University), No.20 Shaoxian Road, Kundulun District, Baotou City 014032, Inner Mongolia, China Full list of author information is available at the end of the article
}

reach $50 \%[2,3]$. Sepsis affects most important organs, such as kidney, heart and lung, mainly by inducing cell injuries and apoptosis [4,5]. Therefore, prevention of cell apoptosis is considered as a promising target for anti-sepsis therapy $[4,5]$. However, molecular mechanism of the cell apoptosis induced by sepsis remains unclear [6], resulting in difficulties in the development of novel therapeutic approaches.

Studies on the molecular pathogenesis of sepsis have revealed a large number of molecular pathways involved in this disease [7]. Understanding the functionality of these molecular players in sepsis provided novel insights 
into the development of targeted therapies [8]. Extensive studies have revealed that non-coding RNAs (ncRNAs), such as long (>200 nt) ncRNAs (lncRNAs) and microRNAs (miRNAs), do not encode protein products but play critical roles in diverse biological processes by regulating protein synthesis [9]. In effect, regulating the expression of critical ncRNA players in sepsis may benefit the treatment of this disease [10]. However, the functions of most ncRNAs remain unclear. LncRNA THRIL (TNFa and heterogenous nuclear ribonucleoprotein L ( $h n R N P L)$ related immunoregulatory LincRNA) is an enhancer of LPS-induced inflammatory [11]. In another study, THRIL was demonstrated to increase the risk of acute respiratory distress syndrome (ARDS) and was positively correlated with inflammatory responses, disease severity and mortality in sepsis patients [12]. Our bioinformatics analysis showed that THRIL may interact with miR-19a, which targets TNF- $\alpha$ [13], a critical player in sepsis [14]. This study was therefore performed to investigate the interactions among THRIL, miR-19a and TNF- $\alpha$ in sepsis.

\section{Methods}

Sepsis patients and healthy controls

Research subjects of the present study included 66 sepsis patients ( 40 males and 26 females, 42 to 66 years old, mean age $54.2 \pm 6.7$ years old) and 66 healthy volunteers (40 males and 26 females, 42 to 66 years old, mean age $54.0 \pm$ 7.0 years old). All participants were enrolled at the Inner Mongolia Baogang Hospital between December 2017 and December 2018. This study was approved by the Ethics Committee of this hospital. No therapy was initiated before the admission of patients. All patients were diagnosed with sepsis for the first time. No previous history of severe diseases, such as cancers, diabetes and heart diseases were observed. All healthy controls were selected at the physiological healthy center of aforementioned hospital after they received systemic physiological examinations. All physiological functions of the healthy controls were normal. All participants signed the written informed consent.

\section{Plasma preparations}

After fasting for overnight, blood extraction $(5 \mathrm{ml})$ was performed on all patients (before the use of antibiotics) and controls. Blood samples were mixed with EDTA and were centrifuged $(1200 \mathrm{x} \mathrm{g})$ at room temperature for $10 \mathrm{~min}$ to separate plasma. Plasma samples were stored in liquid nitrogen.

\section{Human bronchial epithelial cells (HBEpCs)}

All cell experiments were performed using HBEpCs (Sigma-Aldrich). Cell culture was performed following the manufacturers' instructions. All passage 4-6 generations, cells were collected at about $85 \%$ confluence to be used in following experiments.

\section{RNA-RNA interaction prediction}

The potential base paring formed by THRIL and miR19a was predicted by IntaRNA 2.0 (http://rna.informatik. uni-freiburg.de/IntaRNA/Input.jsp). THRIL was used as long sequence and miR-19a was used as short sequence. All other parameters were default.

\section{Transient transfections and dual luciferase reporter assay} Expression vector of THRIL or TNF- $\alpha$ was constructed using pcDNA 3.1 vector (Invitrogen) as the backbone. Negative control (NC) miRNA, miR-19a mimic, miR-19a inhibitor, and THRIL siRNA (siTHRIL) were purchased from Sigma-Aldrich (USA). HBEpCs were transfected with $10 \mathrm{nM}$ expression vector $(10 \mathrm{nM})$ or miRNA mimic $(40 \mathrm{nM})$ and their relevant siRNA or inhibitor using Lipofectamine 2000 (Invitrogen). Untransfected cells were used as the control $(\mathrm{C})$ cells. NC cells were empty vectoror NC miRNA- transfected cells. To perform dualluciferase reporter assay, THRIL luciferase reporter vector was constructed using pmirGLO vector (Promega, USA). HBEpCs were co-transfected with either THRIL vector and miR-19a (miR-19a group) or THRIL vector and NC miRNA (NC group) using lipofectamine 2000. DualLuciferase Reporter Assay System (Promega) was used to measure luciferase activity $48 \mathrm{~h}$ post-transfection.

\section{RNA preparations}

Total RNAs were isolated from HBEpCs and plasma samples using Trizol reagent (Invitrogen), following by digestion with DNase I to remove genomic RNAs. RNA precipitation and washing steps were performed using $85 \%$ ethanol. The RNA concentrations were measured using the Thermo Fisher NanoDrop 2000 Spectrophotometer. To assess the effects of LPS (L2630-25MG, Sigma-Aldrich) treatment on the expression of THRIL, RNA isolation from HBEpCs was performed after treatment with $0,2,5$ or $10 \mu \mathrm{g} / \mathrm{ml}$ LPS for $24 \mathrm{~h}$.

\section{RT-qPCR assay}

BlazeTaq $^{\text {Tit }}$ One-Step SYBR Green RT-qPCR Kit was used to prepare PCR reactions. The expression levels of THRIL and TNF- $\alpha$ were measured with GAPDH as endogenous control. The measurement of the expression of mature miR-19a was performed using All-in-One ${ }^{\mathrm{m}}$ miRNA qRT-PCR reagent kit (GeneCopoeia). All operations were performed following the manufacturers' instructions. PCR reactions were repeated 3 times and the $2^{-\Delta \Delta C T}$ method was used to perform data analysis.

\section{Western blotting}

The isolation of total proteins from HBEpCs was performed using RIPA solution (Sigma-Aldrich), followed by BCA assay to measure protein concentrations. All protein samples were incubated at $95^{\circ} \mathrm{C}$ for $10 \mathrm{~min}$ to denature proteins, followed 
by separating protein samples ( $35 \mu \mathrm{g}$ protein per lane) using $10 \%$ SDS-PAGE gel. PVDF membranes were used to transfer proteins and blocking in 5\% non-fat milk (FBS) at room temperature for $2 \mathrm{~h}$. After that, primary antibodies including rabbit anti-TNF- $\alpha$ (ab9635, Abcam) and GAPDH (ab9483, Abcam) were used to incubate with membranes at $4{ }^{\circ} \mathrm{C}$ for 12 h. After that, HRP Goat Anti-Rabbit (IgG) (ab6721, Abcam) secondary antibody was used to incubate with the membranes at room temperature for $2 \mathrm{~h}$. Data normalization was performed using ImageJ v1.48.

\section{Cell apoptosis assay}

HBEpCs were harvested at $48 \mathrm{~h}$, followed by incubation in medium containing $10 \mu \mathrm{g} / \mathrm{ml}$ LPS for another $48 \mathrm{~h}$. After that, cells were harvested and washed with precold PBS. PI and FITC-annexin V staining were performed and flow cytometry was used to separate apoptotic cells.

\section{Statistical analysis}

Data from 3 independent biological replicates were expressed as mean \pm SEM. Comparisons between two groups were performed using unpaired t test. Comparisons among multiple groups were performed using ANOVA (one-way) and Tukey test. $P<0.05$ was considered to be statistically significant.

\section{Results}

Expression of THRIL, miR-19a and TNF- $a$ were altered in sepsis patients

The expression levels of THRIL, miR-19a and TNF- $\alpha$ in plasma from sepsis patients $(n=66)$ and healthy controls $(n=66)$ were measured using RT-qPCR assay. Compared to the control group, significantly upregulated THRIL was observed in sepsis patients (Fig. 1a, $p<0.05$ ). Moreover, compared to the control groups, sepsis group exhibited significantly lower expression levels of miR-19a (Fig. 1b, $p<$

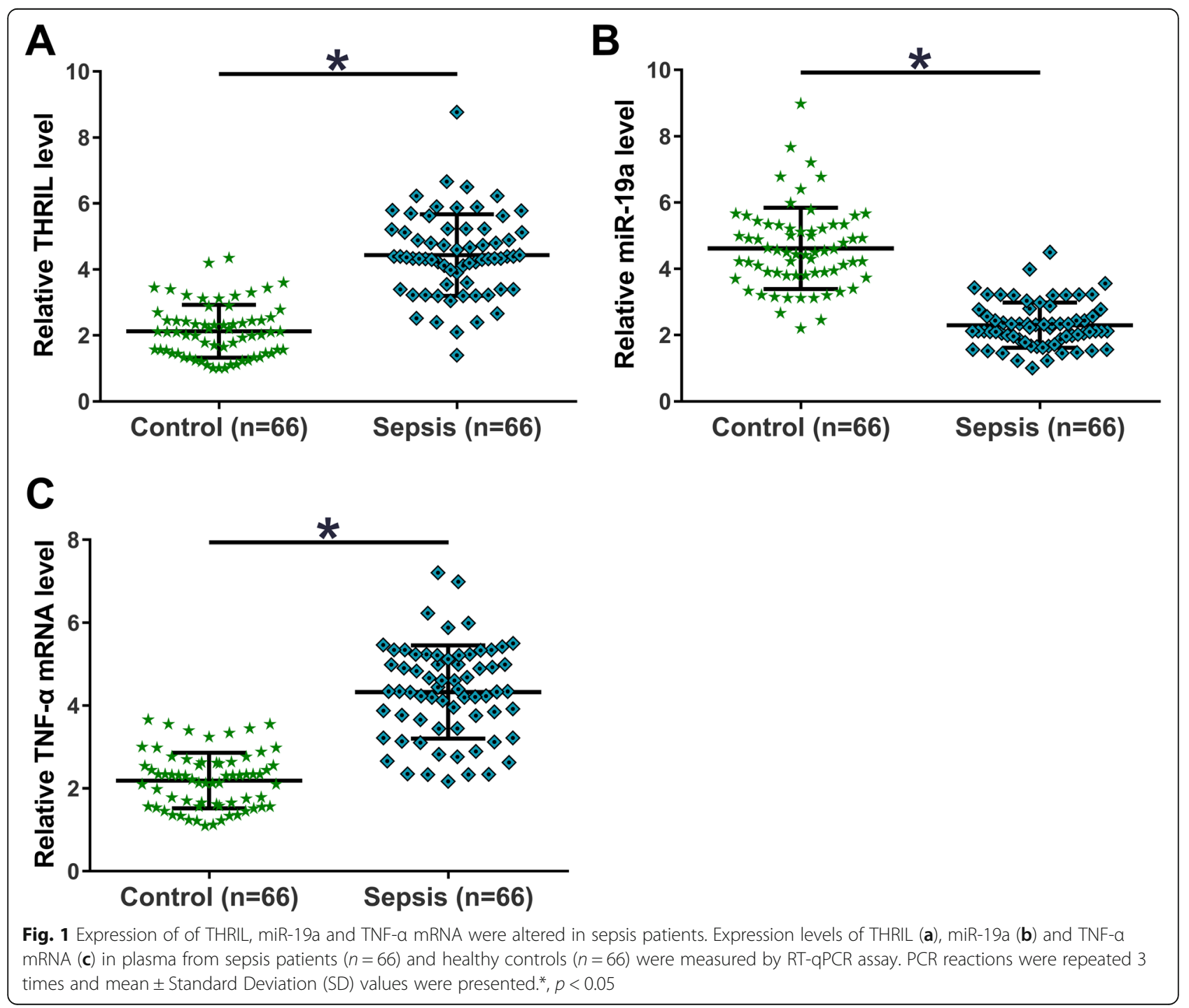


$0.05)$ and significantly higher expression levels of TNF- $\alpha$ (Fig. 1c, $p<0.05$ ). These data suggested that THRIL, miR$19 \mathrm{a}$ and TNF- $\alpha$ may participate in sepsis.

THRIL and miR-19a interacted with each other but did not regulate the expression of each other

The potential interaction between THRIL and miR-19a was predicted by IntaRNA 2.0. It was observed that THRI $\mathrm{L}$ and miR-19a may form strong base pairing (Fig. 2a). Dual luciferase reporter assay was used to evaluate the interaction between THRIL and miR-19a. Compared to NC group (see materials and methods section for grouping details), significantly lower relative luciferase activity was observed in miR-19a group (Fig. $2 b, p<0.05$ ). Therefore, THRIL and miR-19a can directly interact with each other. HBEpCs were transfected with THRIL expression vector or miR-19a mimic to perform further analysis. The overexpression of THRIL and miR-19a was confirmed at $48 \mathrm{~h}$ post-transfection (Fig. $2 \mathrm{c}, p<0.05$ ). Compared to NC and $\mathrm{C}$ groups, overexpression of THRIL and miR-19a did not significantly affect the expression of each other (Fig. 2d). As previous studies showed that THRIL interacts with hnRNPL to regulate TNFa induction, so we further investigated whether THRIL and miR-19a would affect the expression levels of hnRNPL in HBEpCs. However, we found that overexpression of THRIL and miR-19a could not change the expression of hnRNPL (suppl. Fig. 1A-B), which implicated that THRIL or miR-19a have no correlation with the expression of hnRNPL.

\section{Overexpression of THRIL resulted in the increased expression levels of TNF-a}

Data presented above indicated the potential role of THRIL as an endogenous sponge of miR-19a. To test this possibility, the effects of overexpression of THRIL and $\mathrm{miR}-19 \mathrm{a}$ on the expression of TNF- $\alpha$, a downstream target of miR-19a, were analyzed by both RT-qPCR (Fig. 3a) and western blot (Fig. 3b). It was observed that overexpression of miR-19a resulted in downregulated TNF- $\alpha$, further confirming the targeting of TNF- $\alpha$ by miR-19a. In contrast, overexpression of THRIL resulted in upregulated TNF- $\alpha$ and reduced the inhibitory effects of miR-19a on TNF- $\alpha(p<0.05)$.

\section{THRIL regulated the miR-19a/TNF- $a$ axis to promote the apoptosis of HBEpCs}

HBEpCs were cultivated in medium supplemented with $0,2,5$ or $10 \mu \mathrm{g} / \mathrm{ml}$ LPS for $24 \mathrm{~h}$, followed by the
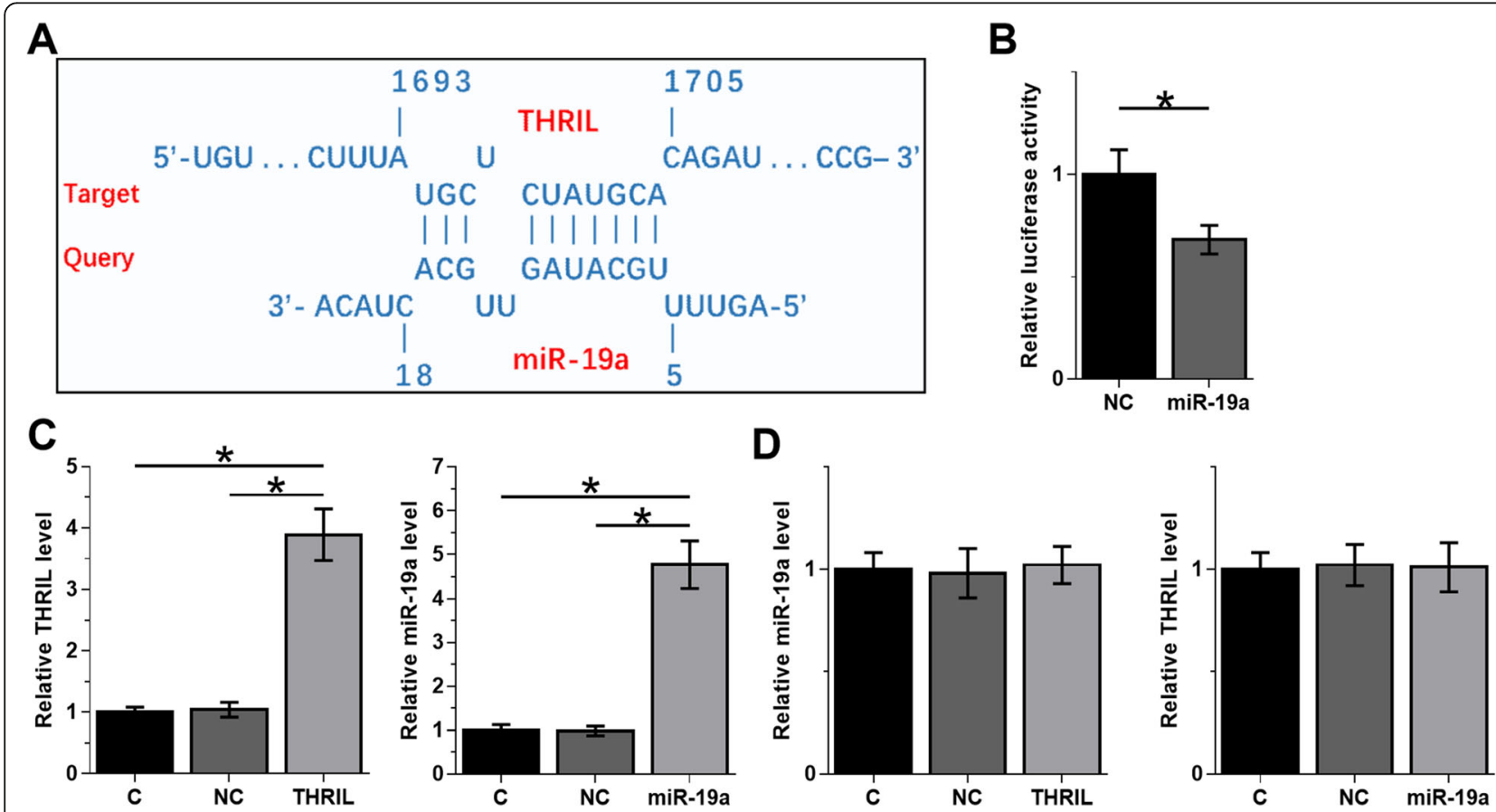

Fig. 2 THRIL and miR-19a interacted with each other but did not regulate the expression of each other. The potential interaction between THRIL and miR-19a was predicted by IntaRNA 2.0. It was observed that THRIL and miR-19a may form strong base pairing (a). Dual luciferase reporter assay was used analyze the interaction between THRIL and miR-19a. HBEpCs were co-transfected with either THRIL vector and miR-19a (miR-19a group) or THRIL vector and NC miRNA (NC group). Luciferase activity was measured at $48 \mathrm{~h}$ post-transfection and was compared between two groups (b). HBEpCs were transfected with THRIL expression vector or miR-19a mimic to explore the interaction between them. The overexpression of THRIL and miR-19a was confirmed at $48 \mathrm{~h}$ post-transfection (c). The effects of the overexpression of THRIL and miR-19a on the expression of each other were also analyzed by RT-qPCR at $48 \mathrm{~h}$ post-transfection (d). All experiments were repeated 3 times and mean \pm SD values were presented. ${ }^{*}, p<0.05$ 
measurement of expression levels of THRIL by performing RT-qPCR. Compared to $\mathrm{C}$ and NC groups, LPS treatment upregulated the expression of THRIL in a dose-dependent manner (Fig. 4a, $p<0.05$ ). Interestingly, we also detected the cell viability after treatment with 0 $20 \mu \mathrm{g} / \mathrm{ml}$ LPS for $24 \mathrm{~h}$ using trypan blue living cell count, cell viability had no significantly change below $10 \mu \mathrm{g} / \mathrm{ml}$ (suppl. Fig. 2) The effects of overexpression of THRIL, miR-19a and TNF- $\alpha$ on the apoptosis of HBEpCs were assessed by cell apoptosis assay. Compared to $\mathrm{C}$ and $\mathrm{NC}$ groups, overexpression of THRIL and TNF- $\alpha$ promoted the apoptosis of HBEpCs induced by LPS, while overexpression of miR-19a inhibited cell apoptosis. Moreover, overexpression of THRIL attenuated the effects of overexpression of miR-19a. However, the effects of THRIL and miR-19a would also be reversed by its relevant siRNA and inhibitor (Fig. 4b-c, $p<0.05$ ).

\section{Discussion}

This study mainly investigated the interactions among THRIL, miR-19a and TNF- $\alpha$ in sepsis. We found that
THRIL was upregulated in sepsis and may upregulate TNF- $\alpha$ by sponging miR-19a to promote cell apoptosis induced by LPS.

THRIL has been proven to participate in multiple pathological processes $[11,15]$. For instance, silencing of THRIL leads to altered expression of miR-99a, which in turn suppresses hypoxia-induced injuries in $\mathrm{H} 9 \mathrm{C} 2$ cells [15]. In a recent study, THRIL was demonstrated to be an enhancer of LPS-induced inflammatory injury in ATDC5 cells mainly by reducing the expression levels of miR-125b [11]. It is well-known that LPS-induced inflammatory can promote the progression of sepsis [16]. Therefore, THRIL may also participate in sepsis. To the best of our knowledge, this study is the first to report the upregulation of THRIL in sepsis. In addition, we also revealed that LPS treatment could upregulate the expression of THRIL in HBEpCs, which in turn promoted the apoptosis of lung cells. Therefore, LPS-induced THRIL may participate in sepsis by inducing cell apoptosis.

It has been reported that miR-19a and CD22 could form a feedback regulation loop to regulate the

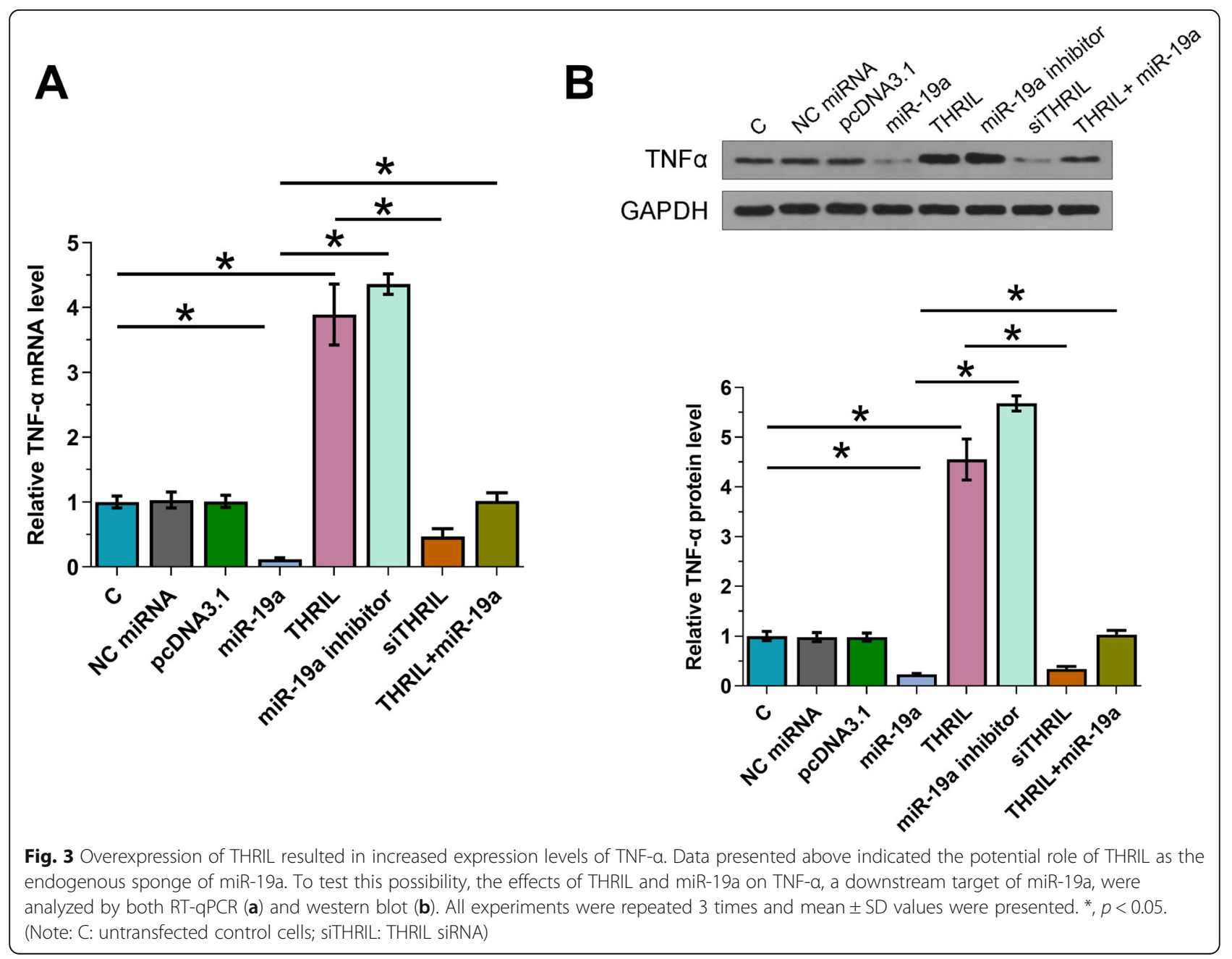




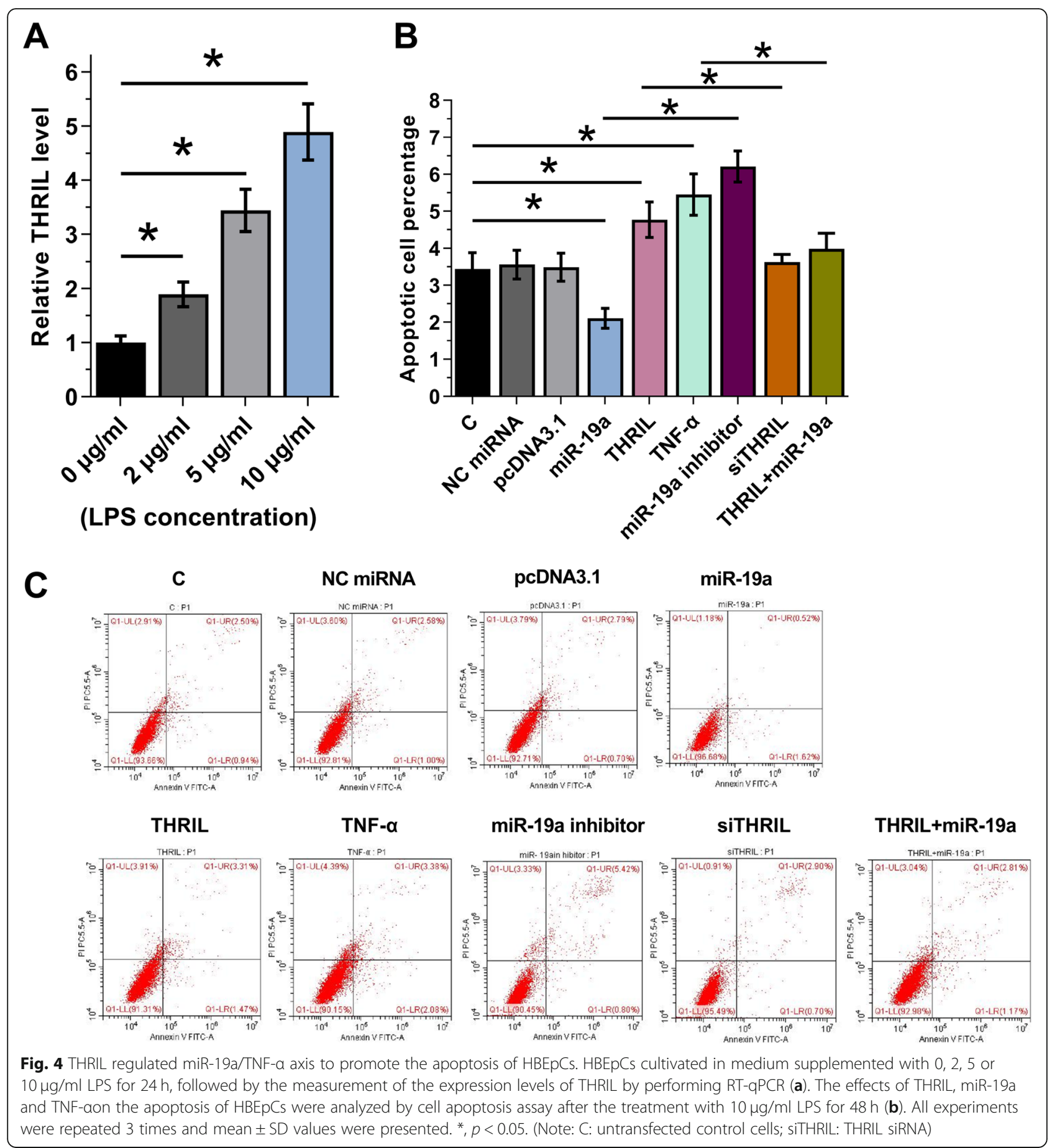

responses of B cells in sepsis [17]. MiR-19a may also participate in sepsis by targeting TNF- $\alpha$ [13], which a critical player in sepsis [14]. Our study confirmed the targeting of TNF- $\alpha$ by miR-19a. In addition, our study is the first to show that miR-19a can target TNF- $\alpha$ to suppress LPS-induced lung cell apoptosis.

The key finding of this study is that THRIL may serve as an endogenous sponge of miR-19a to attenuate its inhibitory effects on cell apoptosis-induced by LPS and the expression of TNF- $\alpha$. Therefore, we characterized a novel THRIL/miR-19a/TNF- $\alpha$ pathway in sepsis. However, it also has been reported that THRIL may also regulate the expression of TNF $\alpha$ by interacting with hnRNPL [18]. Therefore, THRIL may regulate the expression of TNFa through multiple ways.

This study is limited by the small sample size. In addition, in vivo animal model experiments were not performed in this study. Our future studies will include 
more patients and perform sepsis animal model experiments to further confirm our conclusions. Sepsis induces a series of disorders in major organs. The expression of THRIL may also be affected by those complications. Our future studies will explore the effects of sepsis-related complications on the expression of THRIL.

\section{Conclusion}

In conclusion, THRIL is upregulated in sepsis and may sponge miR-19a to upregulate TNF- $\alpha$, thereby promoting cell apoptosis induced by LPS.

\section{Supplementary information}

Supplementary information accompanies this paper at https://doi.org/10. 1186/s12950-020-00259-z.

Additional file 1: Suppl Figure 1. THRIL and miR-19a did not regulate the expression of hnRNPL in HBEpCs. RT-qPCR detect hnRNPL expression after $48 \mathrm{~h}$ transfection of THRIL and miR-19a into HBEpCs, respectively. (A) Overexpression of THRIL could not change the expression of hnRNPL; (B) MiR-19a could not regulate the expression of hnRNPL. (Note: C: untransfected control cells).

Additional file 2: Suppl Figure 2. Lower concentration of LPS would not affect HBEpCs cell viability. The cell viability after treatment with 0$20 \mu \mathrm{g} / \mathrm{ml}$ LPS for $24 \mathrm{~h}$ was analyzed by trypan blue living cell count, cell viability had no significantly change below $10 \mu \mathrm{g} / \mathrm{ml}\left({ }^{*}, p<0.05\right)$.

\section{Abbreviations}

HBEpCs: human bronchial epithelial cells; IncRNA TNFa and heterogenous nuclear ribonucleoprotein $L$ (hnRNPL) related THRIL: immunoregulatory LincRNA; ncRNAs: non-coding RNAs; IncRNAs: long ncRNAs

\section{Acknowledgements}

Not applicable.

\section{Authors' contributions}

TL and JBL: study concepts, study design, literature research, experimental studies, manuscript preparation and editing; $\mathrm{CHT}$ : definition of intellectual content, literature research experimental studies, manuscript preparation and editing; HYW, MW and MYY: literature research, experiments work and manuscript writing. All authors read and approved the final manuscript.

\section{Funding}

Not applicable.

\section{Availability of data and materials}

The datasets used and/or analyzed during the current study are available from the corresponding author on reasonable request.

\section{Ethics approval and consent to participate}

Ethical approval was obtained from the Ethics Committee of Inner Mongolia Baogang Hospital. Written informed consent was obtained from all individual patients included in the study.

\section{Consent for publication}

Not applicable.

\section{Competing interests}

The authors declare that they have no competing interests.

\section{Author details}

${ }^{1}$ Department of Respiratory and Critical Care Medicine, Inner Mongolia Baogang Hospital (The Third Affiliated Hospital of Inner Mongolia Medical University), Baotou 014032, Inner Mongolia, China. ${ }^{2}$ Department of Respiratory Medicine, Hospital of FIRMACO (The Fourth Affiliated Hospital of Inner Mongolia Medical University), Baotou 014032, Inner Mongolia, China.
${ }^{3}$ Department of Nephrology, Inner Mongolia Baogang Hospital (The Third Affiliated Hospital of Inner Mongolia Medical University), No.20 Shaoxian Road, Kundulun District, Baotou City 014032, Inner Mongolia, China. ${ }^{4}$ Department of Dental department, Hospital of FIRMACO (The Fourth Affiliated Hospital of Inner Mongolia Medical University), Baotou 014032, Inner Mongolia, China. ${ }^{5}$ Department of Pharmacy, Hospital of FIRMACO (The Fourth Affiliated Hospital of Inner Mongolia Medical University), Baotou 014032, Inner Mongolia, China.

Received: 23 December 2019 Accepted: 18 August 2020

Published online: 10 September 2020

\section{References}

1. Chen AX, Simpson SQ, Pallin DJ. Sepsis Guidelines. N Engl J Med. 2019; 380(14):1369-71.

2. Moerer O, Schmid A, Hofmann M, Herklotz A, Reinhart K, Werdan K, et al. Direct costs of severe sepsis in three German intensive care units based on retrospective electronic patient record analysis of resource use. Intensive Care Med. 2002;28(10):1440-6.

3. Seymour CW, Cooke CR, Heckbert SR, Spertus JA, Callaway CW, Martin-Gill C, et al. Prehospital intravenous access and fluid resuscitation in severe sepsis: an observational cohort study. Crit Care. 2014;18(5):533.

4. Graetz TJ, Hotchkiss RS. Sepsis: preventing organ failure in sepsis - the search continues. Nat Rev Nephrol. 2017;13(1):5-6.

5. Lin WC, Chen CW, Huang YW, Chao L, Chao J, Lin YS, et al. Kallistatin protects against sepsis-related acute lung injury via inhibiting inflammation and apoptosis. Sci Rep. 2015;5:12463.

6. Lelubre C, Vincent JL. Mechanisms and treatment of organ failure in sepsis. Nat Rev Nephrol. 2018;14(7):417-27.

7. Englert JA, Bobba C, Baron RM. Integrating molecular pathogenesis and clinical translation in sepsis-induced acute respiratory distress syndrome. JCl Insight. 2019;4(2):e124061.

8. Zhou Q, Pan X, Wang L, Wang X, Xiong D. The protective role of neuregulin-1: a potential therapy for sepsis-induced cardiomyopathy. Eur J Pharmacol. 2016;788:234-40.

9. Ho J, Chan H, Wong SH, Wang MH, Yu J, Xiao Z, et al. The involvement of regulatory non-coding RNAs in sepsis: a systematic review. Crit Care. 2016. 20(1):383.

10. Zhang T-N, Li D, Xia J, Wu Q-J, Wen R, Yang N, et al. Non-coding RNA: a potential biomarker and therapeutic target for sepsis. Oncotarget. 2017; 8(53):91765-78.

11. Liu G, Wang Y, Zhang M, Zhang Q. Long non-coding RNA THRIL promotes LPS-induced inflammatory injury by down-regulating microRNA-125b in ATDC5 cells. Int Immunopharmacol. 2019;66:354-61.

12. Wang $Y, F u X, Y u$ B, Ai F. Long non-coding RNA THRIL predicts increased acute respiratory distress syndrome risk and positively correlates with disease severity, inflammation, and mortality in sepsis patients. J Clin Lab Anal. 2019;33(6):e22882.

13. Liu M, Wang Z, Yang S, Zhang W, He S, Hu C, et al. TNF-alpha is a novel target of miR-19a. Int J Oncol. 2011;38(4):1013-22.

14. Lv S, Han M, Yi R, Kwon S, Dai C, Wang R. Anti-TNF-alpha therapy for patients with sepsis: a systematic meta-analysis. Int J Clin Pract. 2014;68(4): $520-8$

15. Xia J, Jiang N, Li Y, Wei Y, Zhang $X$. The long noncoding RNA THRIL knockdown protects hypoxia-induced injuries of $\mathrm{H} 9 \mathrm{C} 2$ cells through regulating miR-99a. Cardiol J. 2019;26(5):564-74.

16. Kim MS, Bae GS, Park KC, Koo BS, Kim BJ, Lee HJ, et al. Myrrh inhibits LPSinduced inflammatory response and protects from cecal ligation and puncture-induced sepsis. Evid Based Complement Alternat Med. 2012;2012: 278718.

17. Jiang $Y$, Zhou H, Ma D, Chen ZK, Cai X. MicroRNA-19a and CD22 comprise a feedback loop for B cell response in Sepsis. Med Sci Monit. 2015;21:1548-55.

18. Li Z, Chao TC, Chang KY, Lin N, Patil VS, Shimizu C, et al. The long noncoding RNA THRIL regulates TNFalpha expression through its interaction with hnRNPL. Proc Natl Acad Sci U S A. 2014;111(3):1002-7.

\section{Publisher's Note}

Springer Nature remains neutral with regard to jurisdictional claims in published maps and institutional affiliations. 